\title{
Subdiffusion and Heat Transport in a Tilted Two-Dimensional Fermi-Hubbard System
}

\author{
Elmer Guardado-Sanchez $\odot{ }^{\dagger}$ Alan Morningstar $\odot{ }^{\dagger}$ Benjamin M. Spar®, Peter T. Brown $\odot$, \\ David A. Huse, and Waseem S. Bakrø" \\ Department of Physics, Princeton University, Princeton, New Jersey 08544, USA
}

(Received 13 September 2019; accepted 20 December 2019; published 21 February 2020)

\begin{abstract}
Using quantum gas microscopy, we study the late-time effective hydrodynamics of an isolated cold-atom Fermi-Hubbard system subject to an external linear potential (a "tilt"). The tilt is along one of the principal directions of the two-dimensional square lattice and couples mass transport to local heating through energy conservation. Because of this coupling, the system quickly heats up to near infinite temperature in the lowest band of the lattice. We study the high-temperature transport and thermalization in our system by observing the decay of prepared initial density waves as a function of wavelength $\lambda$ and tilt strength and find that the associated decay time $\tau$ crosses over as the tilt strength is increased from characteristically diffusive to subdiffusive with $\tau \propto \lambda^{4}$. In order to explain the underlying physics and emphasize its universal nature, we develop a hydrodynamic model that exhibits this crossover. For strong tilts, the subdiffusive transport rate is set by a thermal diffusivity, which we are thus able to measure as a function of tilt in this regime. We further support our understanding by probing the local inverse temperature of the system at strong tilts, finding good agreement with our theoretical predictions. Finally, we discuss the relation of the strongly tilted limit of our system to recently studied 1D models that may exhibit nonergodic dynamics.
\end{abstract}

DOI: 10.1103/PhysRevX.10.011042

\section{INTRODUCTION}

While noninteracting particles in a tilted lattice potential have been studied for almost a century [1-4], the dynamics of strongly tilted and isolated many-body systems with strong interactions have been relatively unexplored. Characterizing the late-time behavior of such closed quantum many-body systems away from equilibrium is a topic of fundamental interest. In a series of recent papers [5-11], it was shown how irreversible dissipative dynamics can emerge from the unitary evolution of closed quantum systems. Thus, generically, we expect the transport of conserved quantities in such systems to behave hydrodynamically at late times as long as the system thermalizes. On the experimental front, advances in quantum simulation with cold atoms and other platforms have allowed for unprecedented control of quantum many-body systems, and for the controlled study of their dynamics [12-18].

\footnotetext{
*Corresponding author. wbakr@princeton.edu

${ }^{\dagger}$ These authors contributed equally to this work.

Published by the American Physical Society under the terms of the Creative Commons Attribution 4.0 International license. Further distribution of this work must maintain attribution to the author(s) and the published article's title, journal citation, and DOI.
}

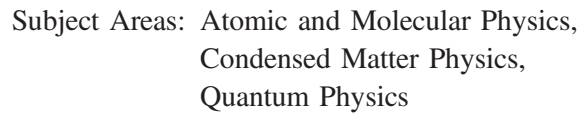

For example, in a recent study, diffusive charge transport was observed in an isolated, strongly interacting, 2D FermiHubbard system [18]. Here, we follow that work by observing the dynamics of the same cold-atom FermiHubbard system subject to a strong external linear potential, or "tilt," and find a crossover to qualitatively different subdiffusive behavior at strong tilts.

The dynamics of a weakly tilted 2D Fermi-Hubbard model were studied in Ref. [19] using semiclassical methods. That work formulated an understanding of the long-time dynamics in which regions with positive local temperature (lower energy and lower entropy than infinite temperature) heat up and transport charge "up" the tilt, and regions with negative local temperature [20,21] (higher energy and lower entropy than infinite temperature) transport charge "down" the tilt as the system approaches an infinite-temperature equilibrium. This infinite-temperature equilibrium of many fermions hopping in a single band has a spatially uniform density and zero expectation value of the hopping energy. In contrast, recent theoretical works $[22,23]$ explored the prospect of a transition to a localized phase in strongly tilted interacting $1 \mathrm{D}$ systems. While some evidence for this transition was found, it was suggested that it was the result of energetically imposed, local kinetic constraints that conserve the center of mass (c.m.) - a phenomenon later referred to as "Hilbert space fragmentation" $[24,25]$. This mechanism for nonergodicity at strong 
tilts depends on factors such as the range of interactions, the dimensionality of the system, and the direction of the tilt. In what follows, we explore a system that does not exhibit such nonergodicity. Thus, this work is most directly related to Refs. $[18,19]$, although initial motivation for this study was derived from Refs. [22,23], and investigating any nonergodic aspects of tilted systems is an interesting avenue for future work.

In this work, we study the effect of an external tilt on the late-time, high-temperature, emergent hydrodynamics of a $2 \mathrm{D}$ cold-atom system. This study is performed by varying the tilt strength and observing the relaxation of prepared initial density waves of various wavelengths $\lambda$. We observe a crossover from a diffusive regime at weak tilts, where the relaxation time $\tau$ scales like $\tau \propto \lambda^{2}$, to a subdiffusive regime at stronger tilts, where $\tau \propto \lambda^{4}$. We then construct a hydrodynamic model that exhibits the same universal crossover and discuss the underlying physics that leads to the subdiffusive transport. Using the hydrodynamic model, we extract the infinite-temperature, tilt-dependent, thermal diffusivity of this system. We further verify our understanding of the underlying physics by measuring the local inverse temperature profile of the system, thus confirming a prediction of our theoretical model that this profile should correspond to local equilibrium and be displaced by a quarter wavelength relative to the density profile.

\section{SYSTEM}

Our system is well described by the tilted FermiHubbard Hamiltonian $\hat{H}=\hat{H}_{\mathrm{FH}}-F \hat{N}_{f} \hat{x}_{\text {c.m. }}$, where $\hat{H}_{\mathrm{FH}}$ is the conventional Fermi-Hubbard Hamiltonian on a square lattice, $F$ is the tilt strength, $\hat{N}_{f}$ is the total number of fermions, and $\hat{x}_{\mathrm{c} . \mathrm{m}}$. is the $x$ component of the c.m. The repulsive on-site interaction energy is denoted by $U$, and the single-particle hopping energy is denoted by $t_{h}$. We emphasize that the system is tilted in only one of the lattice directions, which we denote by $x$. Because of this alignment, transport along the $y$ direction does not couple to the tilt potential. Thus, each row of sites at each $x$ position forms a thermal bath along an equipotential of the tilt. These local baths allow this closed system to thermalize. This case is in contrast to the 1D case for which recent works [22,23] have suggested the possibility of ergodicity breaking in strongly tilted systems.

We realize our tilted 2D Fermi-Hubbard model by loading a balanced mixture of two hyperfine ground states of ${ }^{6} \mathrm{Li}$ into an optical lattice [26]. The tilt is generated by an off-centered 1064-nm Gaussian beam of waist about $180 \mu \mathrm{m}$, as depicted in Fig. 1(a). The gradient of the resulting potential is uniform to within $10 \%$ across a region of length $40 a_{\text {latt }}(30 \mu \mathrm{m})$, where $a_{\text {latt }}$ is the spacing of the optical lattice, and the strength of the potential gradient can be tuned from 0 to about $h \times 5.5 \mathrm{kHz} / a_{\text {latt }}$ [27]. The beam is oriented such that the gradient is aligned with one of the two principal axes of the square lattice. A spatial light modulator (SLM) is used to project sinusoidal potentials of tunable wavelength along the direction of the gradient and also to remove any harmonic confinement from trapping potentials in the region of interest, similar to what was done in Ref. [18]. This method allows us to prepare initial density modulations of tunable wavelength. We also add "hard walls" in the direction perpendicular to the gradient

(a)

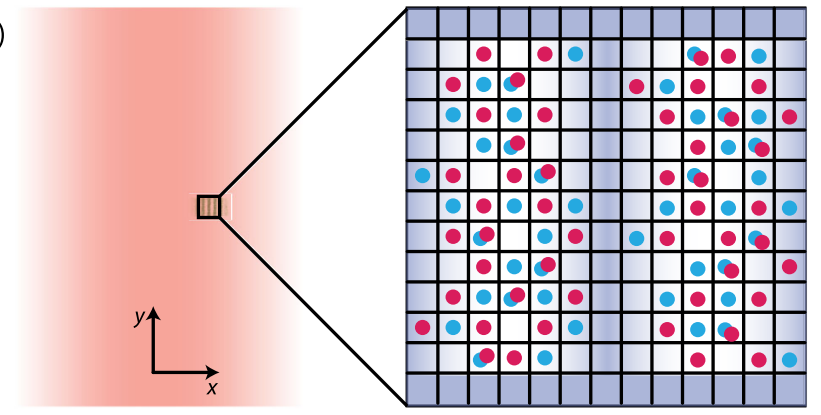

(b)

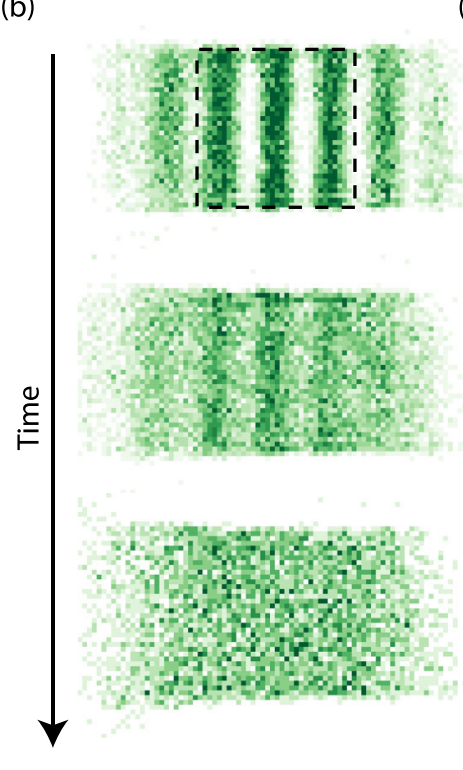

(c)

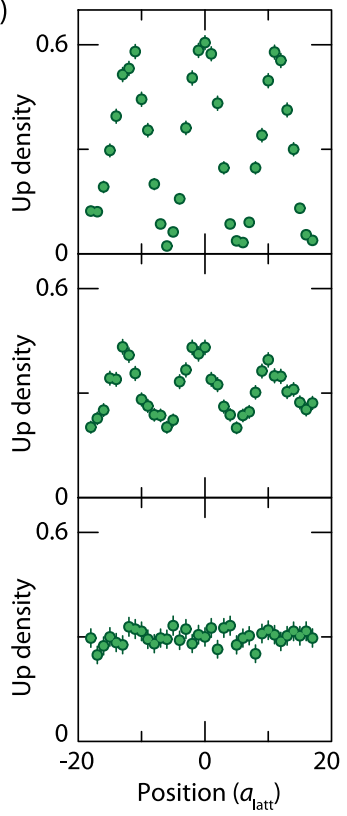

FIG. 1. Experimental setup and measurements. (a) An offcentered beam generates a potential at the atoms that is approximately linear in $x$ and independent of $y$. Blue-detuned light projected through a spatial light modulator is used to prepare the initial density waves of our experiments, with tunable wavelength in the direction of the tilt and hard walls a distance of $35 a_{\text {latt }}$ apart in the perpendicular direction. The figure is a schematic intended to portray the experimental setup and is not to scale. (b) Spin-up ( $\uparrow$ ) component of density vs time, averaged over about 10 images. The dotted square denotes the region of interest (ROI) in which our measurements were taken. (c) Evolution of the $y$-averaged density in the ROI of panel (b) as a function of $x$. The data correspond to a system with interaction energy $U / t_{h}=$ 3.9(1), tilt strength $F a_{\text {latt }} / t_{h}=0.99(3)$, and an initial density modulation of wavelength $\lambda / a_{\text {latt }}=11.46(3)$. The density profile is shown at times $0 \mathrm{~ms}\left(0 \hbar / t_{h}\right), 0.5 \mathrm{~ms}\left(2.6 \hbar / t_{h}\right)$, and $15 \mathrm{~ms}(77$ $\hbar / t_{h}$ ) from top to bottom. 
in order to contain the atoms in that direction and keep the average density constant over the experimental runtime [see Fig. 1(a)].

The atoms are adiabatically loaded into the lattice-plusSLM potential at zero gradient (no tilt). The sinusoidal component of the SLM potential is chosen such that the resulting atom-density wave varies spatially with $0.0 \lesssim$ $\left\langle\hat{n}_{i}\right\rangle \lesssim 1.2$ [see Figs. 1(b) and 1(c)], where $\hat{n}_{i}=\hat{n}_{i, \uparrow}+\hat{n}_{i, \downarrow}$. We also perform experiments with smaller-amplitude density waves and find no qualitative difference in our results [27]. Once the initial density wave is prepared, we suddenly turn off the sinusoidal component of the potential created by the SLM and turn on the tilt potential, thus initiating the dynamics. We focus on a square region of interest with a size of $35 \times 35$ lattice sites and measure only the single spin component $\left\langle\hat{n}_{i, \uparrow}\right\rangle$ using fluorescence imaging [26] since in a spin-balanced system $\left\langle\hat{n}_{i}\right\rangle=2\left\langle\hat{n}_{i, \uparrow}\right\rangle$.

We perform all experiments at an optical lattice depth of 7.4(1) $E_{R}$, where $E_{R} / h=14.66 \mathrm{kHz}$ is the recoil energy and $h$ is Planck's constant. This method leads to a hopping rate of $t_{h} / h=820(10) \mathrm{Hz}$. We work at a magnetic field of 595.29(4) G near a Feshbach resonance centered on $690 \mathrm{G}$. This process leads to a scattering length of 472.0(9) $a_{0}$, where $a_{0}$ is a Bohr radius, which translates to an interaction energy of $U / t_{h}=3.9(1)$ in the Fermi-Hubbard Hamiltonian. We tune the tilt strength $F$ to values of up to $F a_{\text {latt }} / t_{h} \approx 6$, which allows us to explore tilts well above the crossover from diffusive to subdiffusive dynamics.

It is of note that we do not reach tilt strengths so strong that it would be accurate to describe our system over the experimental runtime using an effective Hamiltonian that exactly conserves the c.m. Therefore, we emphasize that this work does not focus on the physics of fractonlike systems with a strictly conserved dipole moment, nor does it explore the possible nonergodic dynamics in such systems, although these topics are an interesting direction for future research [22-25,28-30]. However, our tilted system does show an emergent conservation of the c.m. in the long-wavelength limit, where the potential energy of the tilt dominates the conserved total energy, and we believe this feature to be universal for tilted interacting lattice systems with energy and charge conservation as long as the particles are restricted to a limited set of bands.

\section{RESULTS}

Our experimental protocol consists of preparing initial density waves of various wavelengths in a potential with tilt $F$ and imaging the system's density profile after it has evolved under its own unitary dynamics for some time $t$. We analyze our data by averaging all measurements from a certain wavelength, tilt, and time, and we also average the density in the direction perpendicular to the tilt. This process yields the averaged density profile along the tilted direction as a function of time, as shown in Fig. 1(c). For each wavelength, tilt, and time, we fit the density profile to a sinusoid, $n(x, t)=\bar{n}+A(t) \cos [\phi(t)+2 \pi x / \lambda]$, after adjusting for any small amount of atom loss, with the wavelength being fixed by the fit to the initial profile. We extract both the phase $\phi$ and amplitude $A$ of the sinusoidal fit as a function of time, normalizing the amplitude by its initial value $A(0)$. The main results of this paper are derived from tracking the decay of the amplitude $A(t)$ with time.

Any change in the phase with time is a result of the distance the center of mass "falls down" the tilt as the system heats up in the first band of the lattice potential. More precisely, an initial state with energy density corresponding to a finite temperature in the nontilted FermiHubbard system will evolve down the gradient of the tilted potential. As it evolves, the tilt does work of about $F \Delta x_{\text {c.m. }}$. per particle for a bulk shift of $\Delta x_{\text {c.m. }}$, and this work gets
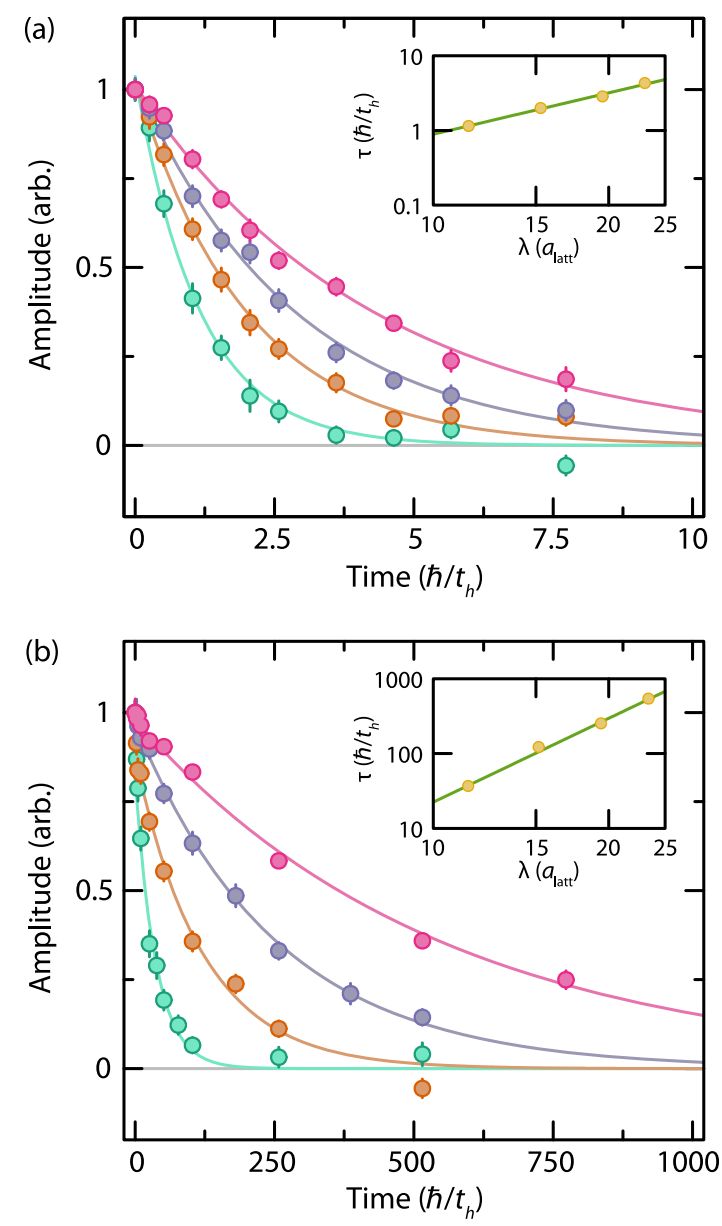

FIG. 2. Time decay of density waves. Fitted normalized relative amplitudes of the periodic density modulation (circles) vs time for wavelengths 11.46(3) (green), 15.16(5) (orange), 19.33(7) (purple), and 23.3(2) (pink) in units of $a_{\text {latt }}$. The lines are exponential fits to the decay at late times after any initial average heating (phase change). (Insets) Log-log plot of the fitted decay times vs wavelength (yellow circles) and a power-law fit of the form $\tau \propto \lambda^{\alpha}$ (green line). (a) Data set for tilt strength $F a_{\text {latt }} / t_{h}=0$. (b) Data set for tilt strength $F a_{\text {latt }} / t_{h}=2.00(3)$. 
converted locally to kinetic and interaction energy in the system (the $t_{h}$ and $U$ terms) [19]. Since the $t_{h}$ and $U$ terms can only accommodate up to an energy of order about $t_{h}+U$ per particle before reaching infinite temperature, the shift of the c.m. of the system cannot be more than about $\left(t_{h}+U\right) / F$. We observe phase changes during the earlytime dynamics that are consistent with this approximate bound [27]. We corroborate that the atoms are not excited to higher bands using a technique described in Ref. [31].

At late times, we observe an approximately exponential decay of the density modulation (see Fig. 2). We fit an exponential to these curves to extract decay times $\tau$ as a function of $\lambda$ and $F$. This process is done at tilts $F a_{\text {latt }} / t_{h} \in\{0,0.39(1), 0.99(3), 2.00(3), 3.88(9), 6.1(2)\}$, and for initial density waves with wavelengths $\lambda / a_{\text {latt }} \in\{11.46(3), 15.16(5), 19.33(7), 23.3(2)\}$. We also use $\lambda / a_{\text {latt }}=7.69(3)$ for $F a_{\text {latt }} / t_{h} \approx 6$ as the decay time of the longest-wavelength modulation becomes very large for this tilt. Decay times that we observe vary increasingly with the tilt strength $F$, from $1-5 \hbar / t_{h}$ at zero gradient up to $10^{3}-10^{4} \hbar / t_{h}$ for $F a_{\text {latt }} / t_{h} \approx 6$. At each value of the tilt strength, we fit a power law of the form $\tau \propto \lambda^{\alpha}$ to our measured decay times. Diffusive relaxation has a characteristic $\tau \propto \lambda^{2}$ dependence $(\alpha=2)$, while values of $\alpha>2$ indicate slower subdiffusive dynamics. Figure 2 shows the full analysis for two of the values of $F$. From the extracted exponents $\alpha$, we observe a crossover from diffusive relaxation at weak tilts, where $\alpha \approx 2$, to subdiffusive behavior with an exponent of $\alpha \approx 4$ at stronger tilts. This

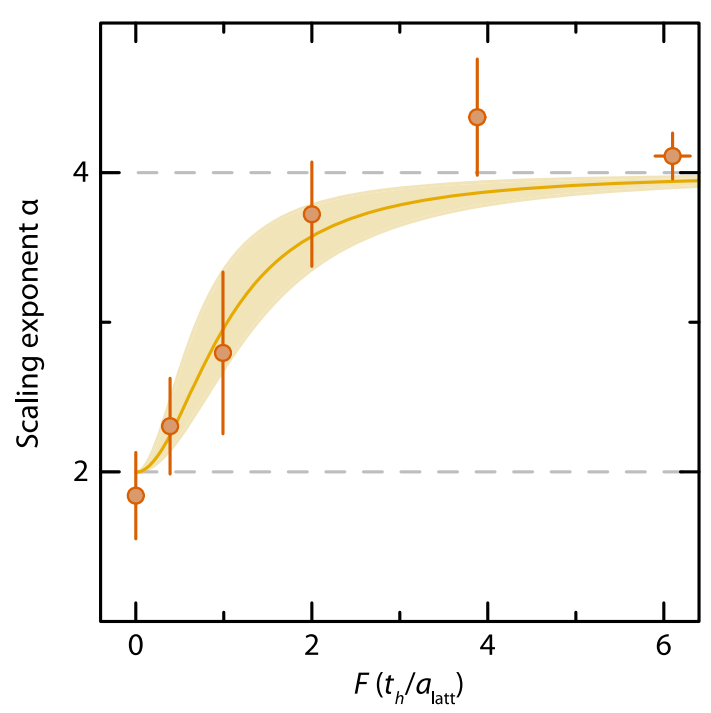

FIG. 3. Diffusive-to-subdiffusive crossover. Extracted scaling exponent $\alpha$ for $\tau \propto \lambda^{\alpha}$ from data sets at different tilts (orange circles). As the tilt is increased from $F a_{\text {latt }} / t_{h}=0$ to $F a_{\text {latt }} / t_{h} \approx 6$, the relaxation of initial density waves crosses over from characteristically diffusive $(\alpha=2)$ to subdiffusive with $\alpha \approx 4$. The shaded curve is a prediction of our hydrodynamic model, which is derived in detail in the Supplemental Material [27]. crossover is shown in Fig. 3, along with the theoretical prediction of our hydrodynamic model.

Our observation of diffusive dynamics at weak tilts is consistent with the analysis of Ref. [19] and with the diffusive transport observed in previous experiments on the same system at $F=0$ [18], albeit at lower temperatures. The crossover to subdiffusion with $\alpha \approx 4$ at strong tilts was, until now, previously unobserved, and its observation and explanation is the main result of this work. Below, and more completely in the Supplemental Material [27], we construct a hydrodynamic model of our system to help explain these observations. We also further test our understanding of the mechanism behind the subdiffusive transport by experimentally verifying our model's predictions for the local temperature profile.

\section{HYDRODYNAMIC MODEL}

We denote the nontilt energy density due to $t_{h}$ and $U$ terms by $e(x, t)$ and the number density of fermions by $n(x, t)$. Our system is, on average, uniform along the $y$ direction, so $e$ and $n$ are assumed to only depend on $x$ and $t$. Here, $n$ is a conserved density and so is $\epsilon=e-F x n$, the total energy density including the tilt potential.

For nonzero tilt, our system heats up to near infinite temperature within the lowest band, where the thermodynamic properties are readily calculated using the hightemperature expansion. There are then three unknown transport coefficients in the most general formulation of our model: Diffusivities for each of the two conserved densities and a thermopower coefficient that might be significant for this system since the energy and atom transport are strongly coupled by the tilt. Our data do not have enough detail to allow us to estimate all three of these transport parameters. However, in the stronger-tilt regime where $\tau \sim \lambda^{4}$, a tilt-dependent thermal diffusivity is the only transport coefficient that enters in the relaxation, and thus this parameter can be determined from our measurements. We therefore present our hydrodynamic model in this strong-tilt regime here and encourage interested readers to see the Supplemental Material [27] for a more detailed presentation of the theory that includes the weaker-tilt diffusive regime.

Let us first consider the infinite-temperature equilibrium that our system thermalizes to at late times. This equilibrium is a limit of zero inverse temperature $(\beta \rightarrow 0)$ and infinite chemical potential $(\mu \rightarrow \infty)$, with a finite spatially uniform $\beta \mu$; we call this equilibrium value $\bar{\beta} \mu$. This uniform equilibrium has atom number density $\bar{n}=2 e^{\overline{\beta \mu}} /\left(1+e^{\overline{\beta \mu}}\right)$ per site and zero expectation value of the hopping kinetic energy (the $t_{h}$ term in the Hamiltonian). It is convenient when separating the energy into tilt and nontilt terms to choose the interaction term at each site to be $U\left[n_{\uparrow}-(\bar{n} / 2)\right]\left[n_{\downarrow}-(\bar{n} / 2)\right]$. This choice amounts to changing the total energy and potential $V(x)$ by constants, so it 
does not change the physics. With this choice, the equilibrium nontilt energy density vanishes: $\bar{e}=0$.

The density profile at finite long times has an additional sinusoidal component: $n(x, t)=\bar{n}+A_{0} e^{-t / \tau} \cos k x$, with $k=2 \pi / \lambda$ (choosing the origin so there is no added phase in the argument of the cosine). In the strong-tilt, long-time, small- $k$ regime we are considering now, this density profile is at local equilibrium with a time-dependent and spatially nonuniform inverse temperature $\beta(x, t)$. We assume the system is also near global equilibrium, so we work to lowest order in $A_{0}$ and $\beta$. Near position $x$, if we have local equilibrium in the tilted potential $V(x)=-F x$ in this high-temperature limit, the density is given by $n(x)=$ $2 e^{\beta(\mu+F x)} /\left(1+e^{\beta(\mu+F x)}\right)$. So, in the long-wavelength limit we are considering here, the density gradient is

$$
\frac{d n}{d x}=F n\left(1-\frac{n}{2}\right) \beta(x) .
$$

For positive $\beta$, the sign of this density gradient is familiar: At equilibrium, the density increases as one goes to lower potential energy since the atoms are favored to sit at lowerenergy positions. At negative temperature for fermions in a band, higher-energy positions are favored instead, so the density gradient is of the opposite sign. Quantitatively, the product $F \beta$ captures how much a system at inverse temperature $\beta$ "notices" the tilt $F$. Thus, indeed, we expect that when the system maintains local equilibrium, $d n / d x \propto F \beta$ holds to leading order near $\beta=0$. It follows that to leading order, the temperature profile is given by $-A_{0} k e^{-t / \tau} \sin k x=$ $F \bar{n}[1-(\bar{n} / 2)] \beta(x, t)$. Using this result, along with a hightemperature expansion to write $e$ as a function of $\beta$ to leading order, we obtain the nontilt energy profile

$$
e(x, t)=\frac{A_{0}}{F}\left[4 t_{h}^{2}+U^{2} \frac{\bar{n}}{4}\left(1-\frac{\bar{n}}{2}\right)\right] k e^{-t / \tau} \sin k x
$$

at local equilibrium to lowest order in $A_{0}$ and $k$. Now that we have determined the profiles of $n$ and $e$ by assuming local equilibrium; next, we consider the dynamics and use energy and number conservation to determine the relaxation time $\tau$. In the regime we are now considering, the ratelimiting bottleneck is the transport of nontilt energy (heat) through the system. This bottleneck limits the rate at which tilt energy can be converted to heat and dissipated to the rest of the system, and thus the rate at which the whole system relaxes.

The relaxation of the number density implies, via the continuity equation for atom number, an atom number current density of

$$
j_{n}(x, t)=\frac{A_{0}}{k \tau} e^{-t / \tau} \sin k x .
$$

This current density flows locally along the tilt direction, locally converting tilt energy to nontilt energy. In addition, there is a heat current $j_{h}(x, t)=-D_{\text {th }} \nabla e(x, t)$ flowing due to the temperature gradients, where $D_{\text {th }}(F)$ is a tiltdependent thermal diffusivity. Conservation of energy is then

$$
\dot{e}=D_{\text {th }} \nabla^{2} e+F j_{n},
$$

showing the contribution of heat diffusion and the conversion of energy from tilt to nontilt due to the atom current $j_{n}$. In the strong-tilt regime we are considering, the two terms on the rhs of Eq. (4) are each much larger in magnitude than the lhs: The motion of the atoms converts tilt energy to nontilt energy, which is dissipated by thermal transport, while the amplitude of the inhomogeneities decays slowly $\left(D_{\mathrm{th}} k^{2} \tau \gg 1\right)$. In this strong-tilt regime, the decay rate is

$$
\frac{1}{\tau}=\frac{D_{\mathrm{th}} k^{4}}{F^{2}}\left[4 t_{h}^{2}+U^{2} \frac{\bar{n}}{4}\left(1-\frac{\bar{n}}{2}\right)\right] \ll D_{\mathrm{th}} k^{2},
$$

and the condition for the validity of this regime is

$$
k^{2}\left[4 t_{h}^{2}+U^{2} \frac{\bar{n}}{4}\left(1-\frac{\bar{n}}{2}\right)\right] \ll F^{2} .
$$

We use Eq. (5) to extract the infinite-temperature thermal diffusivity $D_{\text {th }}(F)$ as a function of tilt strength $F$ in the regime consistent with $\tau \propto \lambda^{4}$ and plot the result in Fig. 4 . From the validity condition of Eq. (6), we can also estimate the location of the crossover shown in Fig. 3. Plugging in the experimental values of $U / t_{h}=4$ and $\bar{n}=0.6$, and any value of $k$ from the experimental range $k a_{\text {latt }} \in[2 \pi / 24,2 \pi / 12]$, we get the condition that $\alpha \approx 4$ when $F a_{\text {latt }} / t_{h} \gg 1$, which is consistent with the data shown in Fig. 3. A more complete model is detailed in

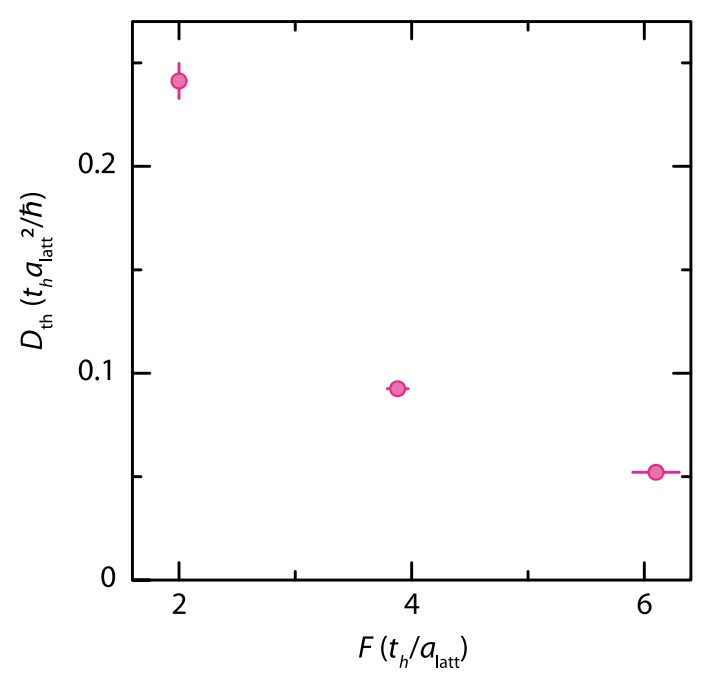

FIG. 4. Thermal diffusivity. Extracted thermal diffusivity (circles) vs gradient. The values are extracted by performing a fit of our hydrodynamic model to all wavelengths of each gradient simultaneously [27]. 

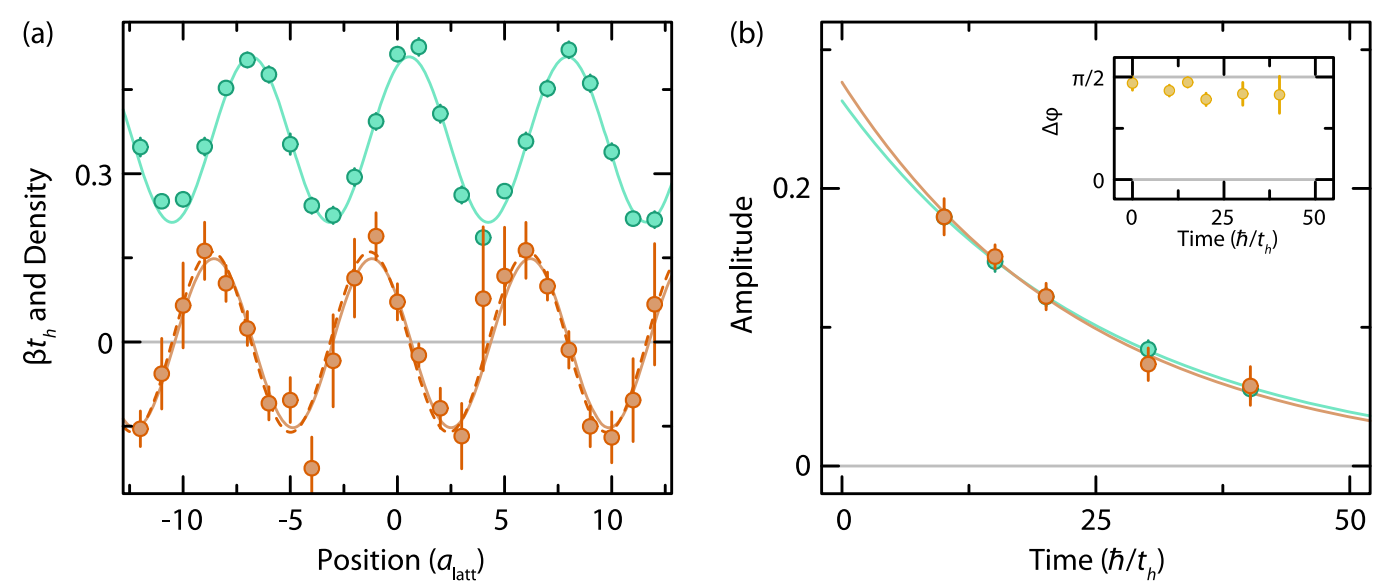

FIG. 5. Local inverse temperature. Near infinite temperature, the density of singles can be used for thermometry. For a tilt strength of $F a_{\text {latt }} / t_{h}=3.4(1)$ [the potential is $V(x)=-F x$ ] and periodic modulation of wavelength $7.69(3) a_{\text {latt }}$, we measure the average singlecomponent density (green) and the density of singles (not shown) in order to extract the local inverse temperature of the cloud (orange). (a) The measured average single-component density (green circles) and extracted inverse temperature $\beta t_{h}$ (orange circles) with sinusoid fits (solid lines) after a decay time of $15.1 \hbar / t_{h}$. In the case of the inverse temperature, the dashed line is the predicted inverse temperature profile from the density fit and local equilibrium [Eq. (1)]. The fitted offset of the inverse temperature is $\bar{\beta} t_{h}=-0.002(8)$, in agreement with an infinite average local temperature. (b) The amplitude of the density (green) and inverse temperature (orange) modulations vs time (circles) with exponential decay fits (solid lines). (Inset) The phase difference of the sinusoid fits between the single-component density and the extracted local inverse temperature vs time (yellow circles).

the Supplemental Material [27], and this model is used to derive the superimposed curve of Fig. 3, which agrees quantitatively with our experimental results. This more detailed model also gives the thermal diffusivity $D_{\text {th }}$ in terms of all of the transport coefficients, including the thermopower. We therefore conclude that our hydrodynamic model captures the essential physics leading to the main observation of this paper: the crossover from diffusive to subdiffusive relaxation with $\tau \propto \lambda^{4}$ as the tilt becomes strong.

The infinite-temperature thermal diffusivity $D_{\text {th }}(F)$ that we are able to measure in this long-wavelength limit is the thermal diffusivity in the presence of a tilt potential and the absence of a mass current. In this long-wavelength limit $(k \rightarrow 0)$, the heat current becomes much larger than the mass current: $j_{h} \sim A_{0} k^{2} / F \gg j_{n} \sim A_{0} k^{3} / F^{2}$. In the limit of small tilt, this thermal diffusivity must be of order $t_{h} a_{\text {latt }}^{2} / \hbar$, with an order-one prefactor that depends on $\bar{n}$ and $U / t_{h}$. In the large-tilt regime where $F a_{\text {latt }} \gg 4 t_{h}$, this heat must be conducted by processes that are second order in the hopping, with one uphill hop and one downhill hop and the intermediate virtual state off shell in energy by $F a_{\text {latt }}$. This method produces an effective matrix element of about $t_{h}^{2} / F$ for these processes, which should result in $D_{\text {th }} \sim 1 / F^{2}$ at large $F$. But our results are actually in an intermediate regime of $F$, where we are able to access this subdiffusive regime, but we are not fully in the large- $F$ regime where one step in the tilt energy is large compared to the interaction $U$ and the bandwidth $4 t_{h}$ for motion along equipotential rows. The results in Fig. 4 seem consistent with matching to these expected small- and large- $F$ limiting behaviors, but we leave a quantitative theoretical estimation of $D_{\text {th }}(F)$ for future work.

The picture we have laid out in this section is one where, at strong tilts and long wavelengths, the system quickly achieves local equilibrium, locking the local inverse temperature to the density profile [Eq. (1)]. As the density profile decays, local number density currents flow, and by conservation of energy, this necessitates the flow of nontilt energy in the system. It is this flow of nontilt energy that bottlenecks the relaxation in the large- $F$ regime, and thus $D_{\text {th }}$ sets the relaxation rate of the system. This mechanism only relies on the fact that the system thermalizes, has a finite maximum kinetic and interaction energy per particle, and obeys energy and charge conservation. Thus, our qualitative conclusions are not specific to the FermiHubbard system we study but can be considered universal. A prediction of this understanding is local equilibrium between $\beta(x, t)$ and $n(x, t)$ summarized in Eq. (1). We verify this prediction by measuring the single-component density and singlon occupancy profiles in our system and by solving for the inverse temperature in the atomic limit, which is an effective method of thermometry at such high temperatures. In Figs. 5(a) and 5(b), we show both the density and local inverse temperature profiles, the decay of both of their amplitudes, and the phase difference between them in time (inset). We see that the $\beta(x, t)$ profile is at local equilibrium near infinite temperature $(\beta=0)$, locked at a quarter-wavelength phase shift from the density profile, and both profiles decay together in time, as predicted by our understanding of the subdiffusive regime of this system. 


\section{SUMMARY AND OUTLOOK}

We studied a new regime of thermalization in a squarelattice cold-atom Fermi-Hubbard system subject to an external linear potential. Our system was effectively closed and evolved under its own unitary dynamics starting from prepared initial density waves of various wavelengths $\lambda$. By observing how the amplitude of these initial density modulations evolved in time, we found two qualitatively different hydrodynamic regimes and a crossover between them: At weak tilts, the system relaxes diffusively, in accordance with previous theory [19] and experiments [18]. At strong tilts, we found a new regime where the system relaxes subdiffusively, with a decay time $\tau$ that scales as $\tau \propto \lambda^{4}$. We argued that this subdiffusive behavior is a result of having to "drain" the large reservoir of tilt energy via the bottleneck of heat transport en route to global equilibrium, and it is captured effectively by a hydrodynamic description, with the system remaining near local equilibrium. To test this understanding, we measured the local temperature profile and indeed found that the system remains near local equilibrium as it relaxes in this subdiffusive regime. In the Supplemental Material, we also develop and present a more complete and detailed hydrodynamic model that quantitatively captures the universal crossover between the diffusive and subdiffusive regimes (Fig. 3). In the strongly tilted regime, we used our model to extract the tilt-strengthdependent thermal diffusivity that bottlenecks the relaxation of the system. One perspective on why this novel subdiffusive regime appears is that, in the strong-tilt and long-wavelength limit, the center-of-mass potential energy is the dominant part of the total energy, so energy conservation becomes an emergent almost-conservation of the center of mass.

In contrast to recent theoretical studies of potential ergodicity breaking in tilted $1 \mathrm{D}$ systems [22,23], in this work we focused on the novel effects of a tilt on the approach to equilibrium in an isolated system that does indeed thermalize. This thermalization was robust because our system had a tilt potential along only one of the two principal axes of the lattice, and the resulting unconstrained motion of atoms in the perpendicular direction produced good thermal baths in each such row of the lattice. To arrest this thermalization more microscopically, one avenue of future exploration will be to apply tilt potentials along both axes of the lattice to suppress such local thermalization.

\section{ACKNOWLEDGMENTS}

We thank Vedika Khemani for helpful discussions. This work was supported by the NSF (Grant No. DMR1607277), the David and Lucile Packard Foundation (Grant No. 2016-65128), and the AFOSR Young Investigator Research Program (Grant No. FA9550-16-10269). W. S. B. was supported by the Alfred P. Sloan Foundation. A. M. acknowledges the support of the Natural
Sciences and Engineering Research Council of Canada (NSERC). D. A. H. was supported in part by the DARPA DRINQS program.

[1] F. Bloch, Über die Quantenmechanik der Elektronen in Kristallgittern, Z. Phys. 52, 555 (1929).

[2] G. H. Wannier, Dynamics of Band Electrons in Electric and Magnetic Fields, Rev. Mod. Phys. 34, 645 (1962).

[3] E. E. Mendez, F. Agulló-Rueda, and J. M. Hong, Stark Localization in GaAs-GaAlAs Superlattices under an Electric Field, Phys. Rev. Lett. 60, 2426 (1988).

[4] P. Voisin, J. Bleuse, C. Bouche, S. Gaillard, C. Alibert, and A. Regreny, Observation of the Wannier-Stark Quantization in a Semiconductor Superlattice, Phys. Rev. Lett. 61, 1639 (1988).

[5] A. Nahum, J. Ruhman, S. Vijay, and J. Haah, Quantum Entanglement Growth under Random Unitary Dynamics, Phys. Rev. X 7, 031016 (2017).

[6] A. Nahum, S. Vijay, and J. Haah, Operator Spreading in Random Unitary Circuits, Phys. Rev. X 8, 021014 (2018).

[7] C. W. von Keyserlingk, T. Rakovszky, F. Pollmann, and S. L. Sondhi, Operator Hydrodynamics, OTOCs, and Entanglement Growth in Systems without Conservation Laws, Phys. Rev. X 8, 021013 (2018).

[8] V. Khemani, A. Vishwanath, and D. A. Huse, Operator Spreading and the Emergence of Dissipative Hydrodynamics under Unitary Evolution with Conservation Laws, Phys. Rev. X 8, 031057 (2018).

[9] T. Rakovszky, F. Pollmann, and C. W. von Keyserlingk, Diffusive Hydrodynamics of out-of-Time-Ordered Correlators with Charge Conservation, Phys. Rev. X 8, 031058 (2018).

[10] S. Gopalakrishnan, D. A. Huse, V. Khemani, and R. Vasseur, Hydrodynamics of Operator Spreading and Quasiparticle Diffusion in Interacting Integrable Systems, Phys. Rev. B 98, 220303(R) (2018).

[11] T. Banks and A. Lucas, Emergent Entropy Production and Hydrodynamics in Quantum Many-Body Systems, Phys. Rev. E 99, 022105 (2019).

[12] F. Meinert, M. J. Mark, E. Kirilov, K. Lauber, P. Weinmann, M. Gröbner, and H.-C. Nägerl, Interaction-Induced Quantum Phase Revivals and Evidence for the Transition to the Quantum Chaotic Regime in 1D Atomic Bloch Oscillations, Phys. Rev. Lett. 112, 193003 (2014).

[13] J.-y. Choi, S. Hild, J. Zeiher, P. Schauß, A. Rubio-Abadal, T. Yefsah, V. Khemani, D. A. Huse, I. Bloch, and C. Gross, Exploring the Many-Body Localization Transition in Two Dimensions, Science 352, 1547 (2016).

[14] H. Bernien, S. Schwartz, A. Keesling, H. Levine, A. Omran, H. Pichler, S. Choi, A. S. Zibrov, M. Endres, M. Greiner, V. Vuletić, and M. D. Lukin, Probing Many-Body Dynamics on a 51-Atom Quantum Simulator, Nature (London) 551, 579 (2017).

[15] S. Scherg, T. Kohlert, J. Herbrych, J. Stolpp, P. Bordia, U. Schneider, F. Heidrich-Meisner, I. Bloch, and M. Aidelsburger, Nonequilibrium Mass Transport in the 1D Fermi-Hubbard Model, Phys. Rev. Lett. 121, 130402 (2018). 
[16] M. Rispoli, A. Lukin, R. Schittko, S. Kim, M. E. Tai, J. Léonard, and M. Greiner, Quantum Critical Behaviour at the Many-Body Localization Transition, Nature (London) 573, 385 (2019).

[17] E. Guardado-Sanchez, .T. Brown, D. Mitra, T. Devakul, D. A. Huse, P. Schauß, and W. S. Bakr, Probing the Quench Dynamics of Antiferromagnetic Correlations in a $2 D$ Quantum Ising Spin System, Phys. Rev. X 8, 021069 (2018).

[18] P. T. Brown, D. Mitra, E. Guardado-Sanchez, R. Nourafkan, A. Reymbaut, C.-D. Hébert, S. Bergeron, A.-M. S. Tremblay, J. Kokalj, D. A. Huse, P. Schauß, and W. S. Bakr, Bad Metallic Transport in a Cold Atom FermiHubbard System, Science 363, 379 (2019).

[19] S. Mandt, A. Rapp, and A. Rosch, Interacting Fermionic Atoms in Optical Lattices Diffuse Symmetrically Upwards and Downwards in a Gravitational Potential, Phys. Rev. Lett. 106, 250602 (2011).

[20] A. Rapp, S. Mandt, and A. Rosch, Equilibration Rates and Negative Absolute Temperatures for Ultracold Atoms in Optical Lattices, Phys. Rev. Lett. 105, 220405 (2010).

[21] S. Braun, J. P. Ronzheimer, M. Schreiber, S. S. Hodgman, T. Rom, I. Bloch, and U. Schneider, Negative Absolute Temperature for Motional Degrees of Freedom, Science 339, 52 (2013).

[22] E. van Nieuwenburg, Y. Baum, and G. Refael, From Bloch Oscillations to Many-Body Localization in Clean Interacting Systems, Proc. Natl. Acad. Sci. U.S.A. 116, 9269 (2019).
[23] M. Schulz, C. A. Hooley, R. Moessner, and F. Pollmann, Stark Many-Body Localization, Phys. Rev. Lett. 122, 040606 (2019).

[24] P. Sala, T. Rakovszky, R. Verresen, M. Knap, and F. Pollmann, Ergodicity-Breaking Arising from Hilbert Space Fragmentation in Dipole-Conserving Hamiltonians, arXiv:1904.04266.

[25] V. Khemani and R. Nandkishore, Local Constraints Can Globally Shatter Hilbert Space: A New Route to Quantum Information Protection, arXiv:1904.04815.

[26] P. T. Brown, D. Mitra, E. Guardado-Sanchez, P. Schauß, S. S. Kondov, E. Khatami, T. Paiva, N. Trivedi, D. A. Huse, and W. S. Bakr, Spin-Imbalance in a 2D Fermi-Hubbard System, Science 357, 1385 (2017).

[27] See Supplemental Material at http://link.aps.org/ supplemental/10.1103/PhysRevX.10.011042 for a description of the experimental methods, analysis, and a more formal derivation of the hydrodynamic model.

[28] S. Pai, M. Pretko, and R. M. Nandkishore, Localization in Fractonic Random Circuits, Phys. Rev. X 9, 021003 (2019).

[29] S. Pai and M. Pretko, Dynamical Scar States in Driven Fracton Systems, Phys. Rev. Lett. 123, 136401 (2019).

[30] R. M. Nandkishore and M. Hermele, Fractons, Annu. Rev. Condens. Matter Phys. 10, 295 (2019).

[31] P. T. Brown, E. Guardado-Sanchez, B. M. Spar, E. W. Huang, T. P. Devereaux, and W. S. Bakr, Angle-Resolved Photoemission Spectroscopy of a Fermi-Hubbard System, Nat. Phys. 16, 26 (2020). 